\title{
Lessons From Managing the Breast Malignant Adenomyoepithelioma and the Discussion on Treatment Strategy
}

\author{
Zhu Yuan ${ }^{\mathrm{a}, \mathrm{c}}$, Xiang Qu ${ }^{\mathrm{a}}$, Zhong-Tao Zhang ${ }^{\mathrm{a}}$, Wen G. Jiang ${ }^{\mathrm{b}, \mathrm{c}}$
}

\begin{abstract}
This study set out to investigate the clinical diagnosis and treatment strategies for malignant breast adenomyoepithelioma (AME), thus increasing the clinical knowledge on such disease. Two patients with malignant breast AME in Beijing Friendship Hospital were selected for study. Here we report the diagnosis and treatment processes in terms of the failure experience and lessons and relate our findings to those in the literature. Malignant breast AME is inclined to affect the areola area. It is recommended to conduct simple mastectomy combined with sentinel lymph node dissection due to the low sensitivity of the preoperative imaging diagnosis and difficulty in the pathological diagnosis. Malignant breast AME features strong invasiveness and vulnerability to recurrence and metastasis. Therefore, the operative schemes and clinical treatment strategies should be formulated based on the comprehensive analyses of the physical signs, imageological examinations and pathology.
\end{abstract}

Keywords: Breast; Malignant adenomyoepithelioma; Failure case; Diagnosis and treatment strategies

\section{Introduction}

The glandular epithelium and myoepithelium of the breast simultaneously meet with the neoplastic proliferation which forms the lump with the significant realm, namely, adenomyoepithelioma (AME); such two kinds of lumps separately or simultaneously will undergo malignant transformation form-

Manuscript submitted June 23, 2017, accepted June 27, 2017

aDepartment of General Surgery, Beijing Friendship Hospital, Capital Medical University, Beijing 100050, China

${ }^{\mathrm{b}}$ Cardiff China Medical Research Collaborative, Cardiff University, School of Medicine, Heath Park, Cardiff, CF14 4XN, UK

${ }^{\mathrm{c} C}$ Corresponding Author: Wen G. Jiang, Cardiff China Medical Research Collaborative, Cardiff University, School of Medicine, Heath Park, Cardiff, CF14 4XN, UK. Email: jiangw@cf.ac.uk; Zhu Yuan, Department of General Surgery, Beijing Friendship Hospital, Capital Medical University, Beijing 100050,China. Email: yuzhdoctor@126.com

doi: https://doi.org/10.14740/wjon1055e ing the malignant AME. Cases of malignant breast AME are relatively rare and tend to be reported separately, so that there are no relevant diagnosis and treatment guidelines and mature clinical experience for reference. Two patients with malignant breast AME were admitted to the General Surgery Department of Beijing Friendship Hospital, Capital Medical University and this study summarized the failure, experiences and lessons learned from the diagnosis and treatment processes. The report is presented as follows.

\section{Case Reports}

\section{Case 1}

The patient, female, 58 years old, was admitted to the hospital for 7 days due to a left breast neoplasm on September 21,2011 . On physical examination, an approximately $3 \times 3$ $\mathrm{cm}$ neoplasm was palpable behind the areola in the 9 o'clock direction of the left breast with a pliable but strong texture, clear border, poor activity and seemed to be adherent to the skin. On core needle biopsy pathology, the glandular tube and obvious cell nests under the extrusion were observed, thus it was recommended to conduct intraoperative frozen pathology. On September 27, 2011, the patient underwent extended resection of the left breast neoplasm, a $3.0 \times 2.5 \mathrm{~cm}$ cyst-solid neoplasm. On frozen pathology, the proliferation of fibrous tissue and epithelial cell nests with large-scaled lesions were found and cells were slightly atypical with some glandular structures. After repeated sampling and immunohistochemical examinations, paraffin pathology examination was carried out, showing a malignant AME in the left breast. Immunohistochemical examination showed ER (-), PR (1\% weakly +), Ki-67 $(30 \%+)$, C-erbB-2 (+), CK5/6 (+), p63 $($ partially + ) and $\mathrm{CD}($ partially +$)$. The hospital contacted the patient and made repeated communications to recommend undergoing radical resection of the left breast cancer, but the patient refused.

On August 20, 2012, the patient was hospitalized again for 1 month due to ulceration of the left breast. On physical examination, an approximately $7 \times 6 \mathrm{~cm}$ neoplasm was palpable in the left internal mammary area and fixed on the chest wall. The skin ulceration was found in the central area with about $5 \times 3.5 \mathrm{~cm}$ anabrosis, deep up to the pectoralis, the surface of 


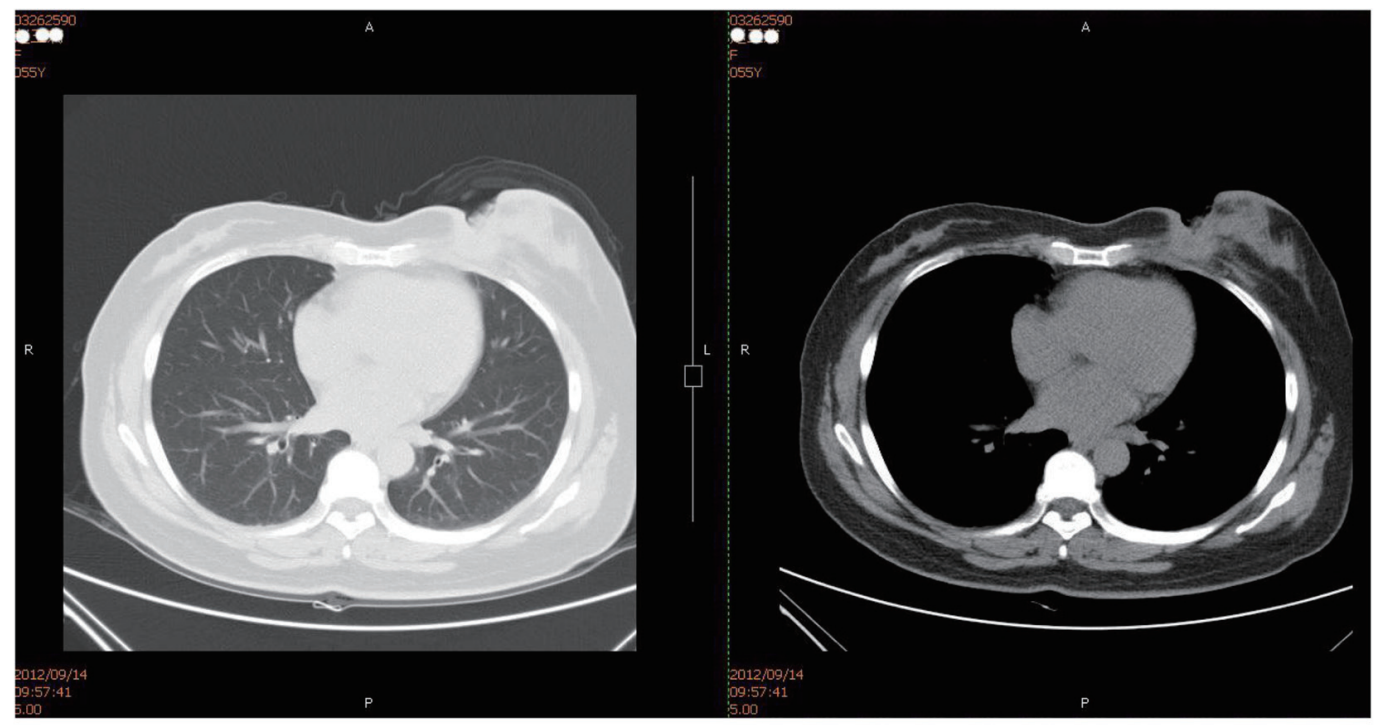

Figure 1. Chest CT (on Setpember 14, 2012): local soft tissues of the left breast were absent and the local skin incrassation was obvious. Subcutaneous irregular soft tissue density image was seen, the border of which with the residual mammary tissue was unclear and stretched inwards the muscle tissues and costal cartilages without definite damage in local rib sclerotin.

which was covered by yellow pus embryo; an approximately $2 \times 1 \mathrm{~cm}$ swollen lymph node was palpable in the left armpit. Chest CT showed the possibility of local recurrence or metastasis (Fig. 1). Biopsy pathology of the anabrosis showed cancer infiltration. Core needle pathology of axillary lymph nodes showed cancerometastasis. The pus embryo bacteria at the anabrosis were cultured into Pseudomonas aeruginosa. No metastasis signs were seen by SPECT-bone scan.
The chemotherapy of TEC (docetaxel + epirubicin + cyclophosphamide) was carried out for two rounds but the tumor did not shrink significantly. After the patient received the treatment of anti-infection and local and debridement, the pus embryo disappeared and no definite pathogenic bacteria were found during the repeated bacterial culture. However, the anabrosis did not shrink. On October 16, 2012, the radical operation of the left breast was performed successfully. On paraffin

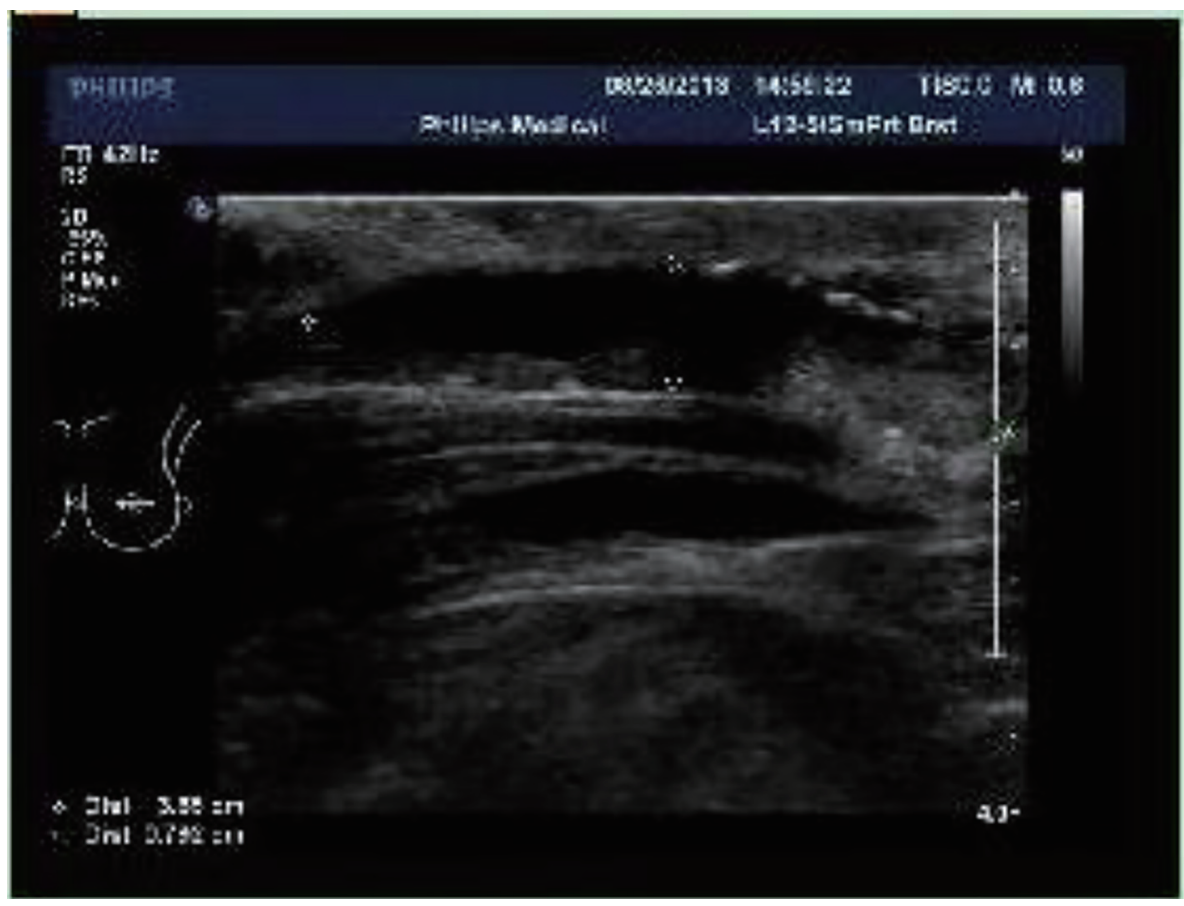

Figure 2. Chest wall Color Doppler ultrasound (on September 6, 2013): multiple flaky echoless region was observed below the incision of the left chest wall with the size of about $3.9 \times 0.8 \mathrm{~cm}$. 


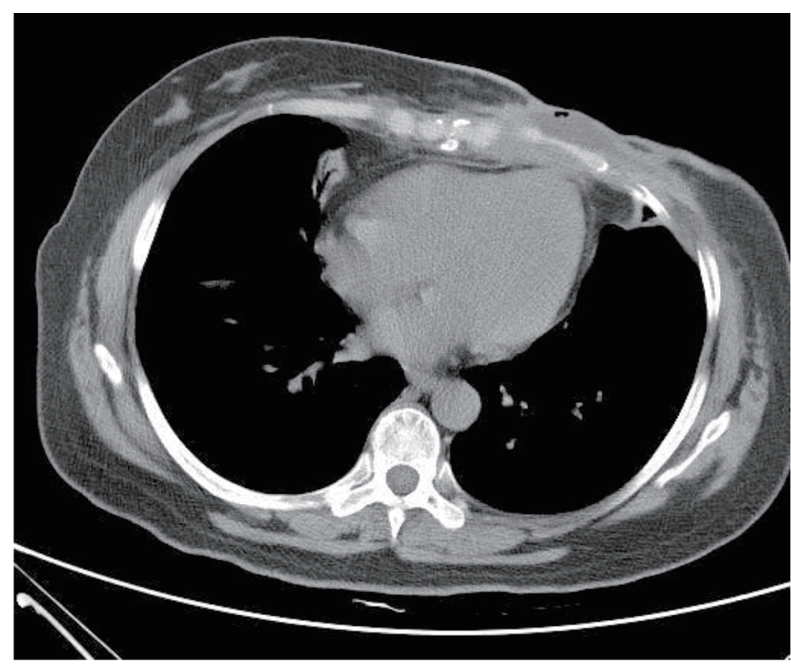

Figure 3. Chest CT (on December 19, 2013): irregular low-density lesions were seen in front of sternum with the maximum cross-section of $4.3 \times 2.9 \mathrm{~cm}$. The border with the incrassated skin and the chest wall were not clear and the bone destruction was found in the sternum and the left fifth rib with the vague edge seen in part of costal cartilages.

pathology, a malignant AME was found in the left breast and the tumor had infiltrated into the skin and pectoralis with the distance from the deep resection margin $<0.1 \mathrm{~cm}$. No tumor embolus was seen in the vessel and 1/12 cancer cells of axillary lymph nodes had metastasized. Immunohistochemical examination showed ER (15\% weakly +), PR (-), Ki-67 (30-40\% + ), C-erbB-2 (+), CK5/6 (+), p63 (partially +), CD10 (partially + ), calponin (partially + ) and CK8 $(+)$. Auxiliary radiotherapy and chemotherapy were carried out. It was found that by the follow-up visit, the intractable subcutaneous hydrops appeared immediately after the operation (Fig. 2). According to the chest $\mathrm{CT}$, bone destruction was seen in the sternum and the left fifth rib (Fig. 3). The patient died from pleural effusion and respiratory failure on August 23, 2014.

\section{Case 2}

The patient, female, 51 years old, was admitted to the hospital on October 14, 2009, 2 weeks after the right breast neoplasm was found. On color Doppler ultrasound, two approximately $1.5 \times 1.0 \mathrm{~cm}$ cyst-solid space-occupying lesions were seen near to the nipple in the 9 o'clock direction of the right breast and BIRADS-US was grade 4. On physical examination, an approximately $3 \times 2 \mathrm{~cm}$ dumbbell-shaped neoplasm was palpable behind the areola area in the 9 o'clock direction of the right breast with a pliable but strong texture, clear border and satisfactory activity. On October 17, 2009, the excision was conducted at the right breast section and frozen pathology was carried out, showing papilloma in the right breast duct. On paraffin pathology, two cyst-solid lesions were observed in the section (diameter: 1.2 and $1.4 \mathrm{~cm}$ ) with the papilloma in the peripheral duct of the right breast. After the operation, the patient recovered well and the follow-up visits were carried out regularly.

On September 17, 2014, the patient was hospitalized again 1 month after the right breast neoplasm was found. On physical examination, an approximately $3.0 \times 2.0 \mathrm{~cm}$ neoplasm was palpable behind the areola in the 9 o'clock direction of the right breast with a pliable but strong texture, clear border, poor activity which seemed to be adherent to the skin. On color Doppler ultrasound, the cyst-solid space-occupying lesion was

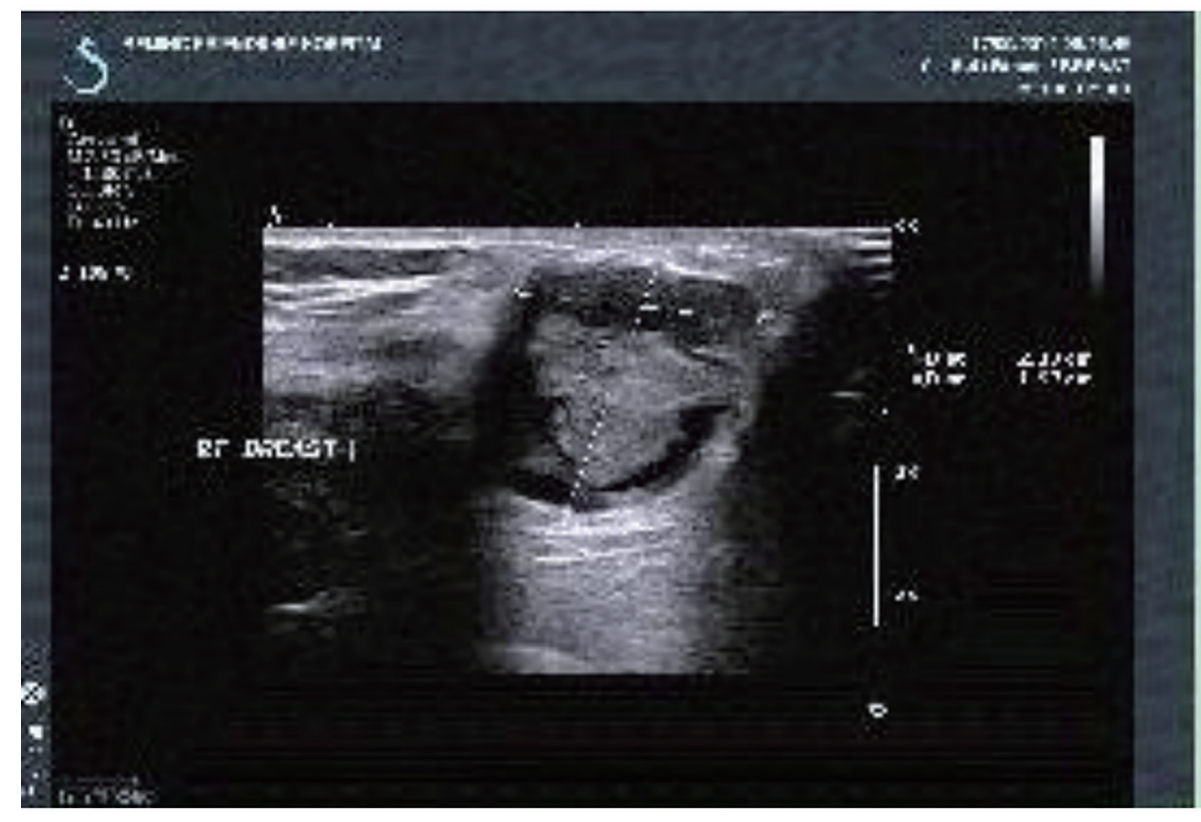

Figure 4. Breast color Doppler ultrasound (on September 17, 2014): a $2.1 \times 2.0 \mathrm{~cm}$ cyst-solid space-occupying lesion was seen near to the nipple in the 9 o'clock direction of the right breast with the clear border and irregular form. Furthermore, the blood flow signal was found with the BI-RADS grading of $4 \mathrm{a}$. 


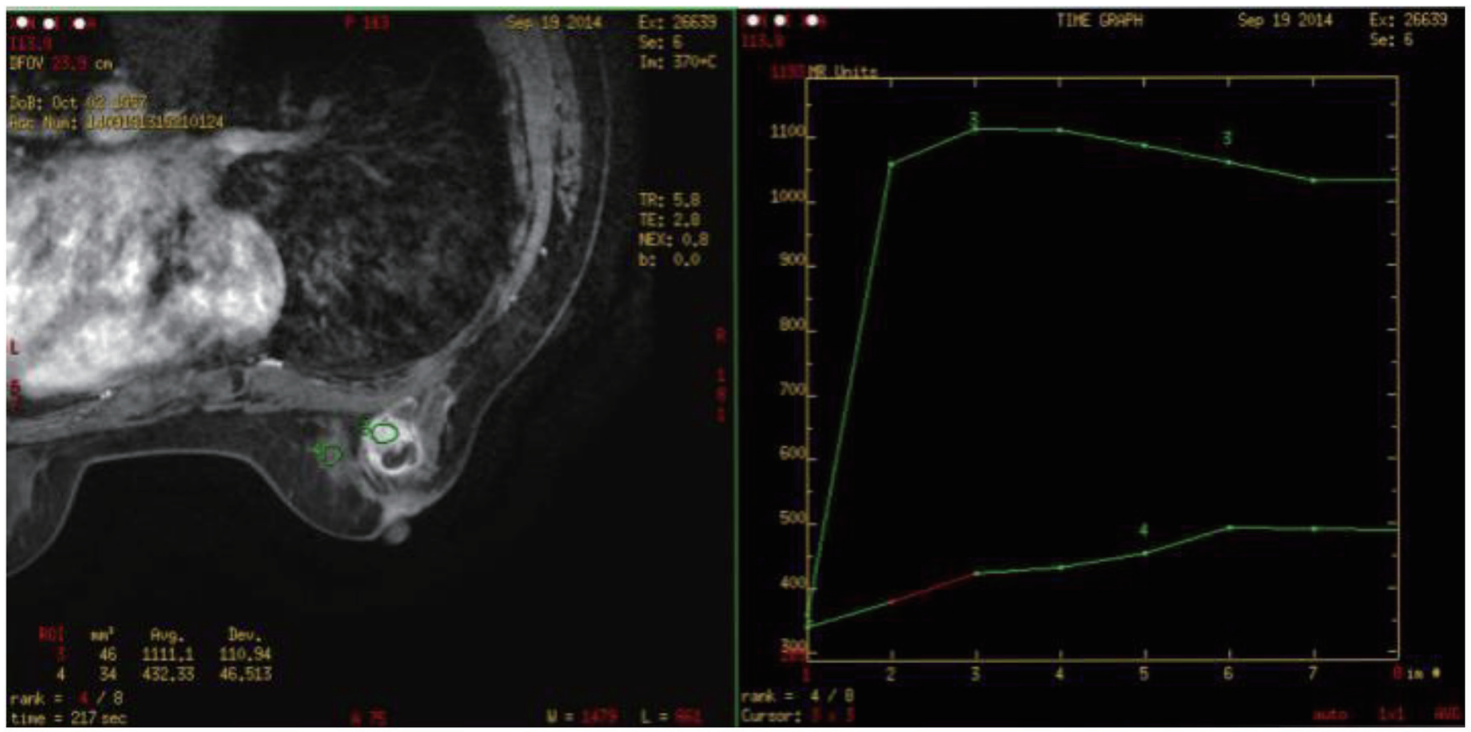

Figure 5. Breast MRI (on September 19, 2014): one about $2.3 \times 1.8 \times 2.8 \mathrm{~cm}$ abnormal signal image with the irregular form was observed in the outer quadrant of the right breast with the equal T1 and long T2 signals. The border was unclear with the burr at the margin. The near skin incrassated and the nipple was inverted. What's more, the border of local lesion with the pectoralis major was unclear. SI-TIME curve: the curve in the high-signal area of the lesion featured type II.

found in the 9 o'clock direction of the right breast with the BI-RADS of grade 4 (Fig. 4). On breast MRI, an abnormal signal was found in the right breast which may be the malignant tumor (Fig. 5).

On core needle biopsy pathology, the ductal epithelial and myoepithelial proliferation was found with atypical hyperplasia of part of the ductal epithelia. On September 20, 2014, the extended resection for the right breast neoplasm was carried out to entirely remove the neoplasm and the adjacent pectoralis major and fascia. On frozen pathology, one cyst-solid neoplasm with the diameter of $1.5 \times 1.5 \mathrm{~cm}$ was found, diagnosed as a papilloma in the right breast duct. After a repeated sampling and immunohistochemical examination, a paraffin pathology examination was carried out, showing the small lesions (two or above will lead to ductal necrosis; the maximum diameter $<2 \mathrm{~mm}$ ) were close to ductal carcinoma in situ; the tumor did not invade the pectoralis major or the fascia. Immunohistochemical examination showed ER (-), PR (-), C-erbB-2 (+), Ki-67 (20\%), E-cadherin (+), CK8 (+), p63 (myoepithelium +) and CK5/6 (+/-). Pathology consultation in another hospital showed AME canceration (myoepithelial carcinoma/malignant adenomyoepithelioma) and part of canceration in the duct. The pathology specimen taken on October 17, 2009 was re-sampled and a review was conducted; finally, the diagnosis results were right breast myoepithelioma.

On October 20, 2014, the patient underwent simple mastectomy of the right breast plus sentinel lymph node biopsy (SLNB) with satisfactory results. On postoperative pathology, the original lesions were excised without the residual; a highly differentiated infiltrating lesion of the ductal carcinoma (grade 1,5 points) with a diameter of $1 \mathrm{~cm}$ was seen in the 3 o'clock direction of the right breast with $0 / 5$ cancer metastasis of the sentinel LN. Immunohistochemical examination showed ER
(80\% strongly +), PR (-), C-erbB-2 (+), Ki-67 (5\%), E-cadherin (+), P120 (membrane +), CK8 (+), CK5/6 (-), p63 (-), CD10 $(-)$, GFAP (-) and calponin (-). The auxiliary postoperative chemotherapy EC (epirubicin + cyclophosphamide) combined with sequential $\mathrm{T}$ (Taxol) was conducted for eight rounds. At the same time, the patient underwent endocrinotherapy. After close follow-up visits over 21 months, the general condition of the patient was satisfactory.

\section{Discussion}

\section{Histologic features}

The malignant breast AME may occur alone, with part of such a tumor becoming malignant from the AME by combining with the infiltrating ductal carcinoma [1]. The malignant AME and infiltrating ductal carcinoma lesions respectively were found at the ipsilateral breast of the second patient as stated, which is consistent with the literature [2].

\section{Immunophenotyping}

The malignant AME may express myoepithelial markers of the basal cells to varying extents such as CK5/6, p63 and SMA, other than ER, PR and Her-2, thus the phenotype features the basal-like breast carcinoma [3]. Two cases in the study group both exhibited the above feature: the first case: ER (-), PR( $1 \%$ weakly +), Ki-67 (30\% +), C-erbB-2 (+), CK5/6 (+), p63 (partially + ) and $\mathrm{CD}($ partially + ); the second case: ER (-), PR (-), C-erbB-2 (+), Ki-67 (20\%), E-cadherin $(+)$, CK8 (+), p63 (myoepithelium + ) and CK5/6 (+/-). 


\section{Predilection site of tumor}

There are definite literature reports on the predilection site of tumor at home, the reason for which may be that the pathologists frequently reporting such cases pay more attention to the histology and immunohistochemical examination of such rare cases. The tumors of two cases in another study group initially occurred in the areola area of five cases reported by Pei et al [4], with these two cases conforming to the above, while Yang et al [5] reported one case. The author made the analysis that this phenomenon may be correlated to the histogenesis of malignant AME, because the myoepithelial cells usually lie between the breast ducts and the basal layers of acinus and the major breast ducts aggregate in the areola area with abundant myoepithelial cells.

\section{Sensibility of preoperative imaging diagnosis}

The initial color Doppler ultrasound examination results of the two cases in the group both showed the cyst-solid neoplasm in the areola area with the BI-RADS grading of 4a. According to the suggestions from Guideline and Specification for the Diagnosis and Treatment of Breast Cancer (version 2015) by Chinese Anti-Cancer Association, grade 4a lesion tends to be benign with the conformity rate to the malignancy of $2-10 \%$ [6]. In terms of the second case in the group, the preoperative MRI showed that the border of local lesions with the pectoralis major was unclear (Fig. 5), while the postoperative pathology certified that the tumor did not invade the pectoralis major and fascia. Therefore, the author believed that the preoperative imaging diagnosis of such case featured the low sensitivity and the physicians with rich clinical experience shall make comprehensive analysis on the patient signs, imaging and pathology for the definite diagnosis without the missed diagnosis.

\section{Preoperative pathological diagnosis}

With the limitation of the rarity and lack of the relevant experience, it is difficult to specify the nature by the intraoperative frozen pathology [7]. However, the complete and accurate pathological diagnosis is the important premise and foundation for the selection of operation methods and clinical treatment, thus it is required to flexibly select the neoplasm biopsy methods. A few tissues of two cases in the paper were obtained through the core needle aspiration biopsy, thus the pathogenesis failed to be identified; instead, the excisional biopsy was carried out and the specific pathological diagnosis was obtained before the operation through the repeated sampling, section, immunohistochemical test and pathology consultation.

\section{Operation methods of breast}

The domestic scholars did not provide the definite conclusion on the selection of breast operation methods. Some scholars have suggested conducting the simple mastectomy or extended resection or breast-conserving surgery on the foundation that safe resection margin will be obtained and local radiotherapy will be conducted flowingly. For the first patient in the paper, the pathogenesis was clear through the locally extended resection, but the simple mastectomy was not operated. It turned out that the patient suffered from the local recurrence and the skin ulceration 11 months after the operation. The patient died from the comprehensive recurrence of the chest wall and bone metastases 10 months after the radical mastectomy of the right breast. For the second patient, the local recurrence and malignant transformation appeared and the infiltrating ductal carcinoma was combined 5 years after the segment resection of the AME but there were no positive signs in the palpationnegative lesions during the preoperative color Doppler ultrasound and MRI examinations. The disease features strong local invasiveness and inclination to recurrence and metastasis and tends to combine with the infiltrating ductal carcinoma and other lesions which cannot be found by the imageological examination. If the pathological diagnosis is clear, the author recommends the simple mastectomy rather than local extended resection or breast-conserving surgery.

\section{Operation method of regional lymph nodes}

A few lymph nodes of malignant AME metastasized. Ahmed et al [8] made the close follow-up visits to 12 cases with the bi-differentiation, where the pulmonary metastasis appeared in three cases and brain metastasis in two cases without none of cases with lymphatic metastasis. Pei et al [4] reported five patients with the malignant AME which all underwent the axillary lymph nodes dissection without the lymph node metastasis. Of two cases with the comprehensive recurrence of the chest wall and bone metastases after the operation, one patient died, but no axillary lymph node metastasis was found in the close follow-up visit. The first patient in the paper suffered from the recurrence and skin ulceration after the local extended resection and there was 1/12 the axillary LN cancerometastasis after the radical resection of the left breast cancer. The second patient underwent the SLNB with 0/5 cancerometastasis. Further discussions are required to specify whether it is applicable to conduct the SLNB without the axillary lymph nodes dissection. What's worse, there are definite conclusions in the literatures at home and abroad. The author thinks that the simple mastectomy combined with SLNB is the preferred operation method.

\section{Adjuvant systemic treatment}

The hematogenous metastasis of malignant AME is common including the liver, brain, bone, and thyroid metastases and the prognosis of patients is extremely poor with the metastasis [9, 10]. Bult et al [11] reviewed such case and found that one-third patients suffered from distant metastases and local recurrence which tend to occur 4 months to 2 or 3 years after the first diagnosis. Based on the features of strong invasiveness and tendency to recurrence and metastasis, the scholars at home and abroad all recommended the adjuvant chemotherapy. Be- 
cause the ER and PR tend to be negative, the endocrinotherapy was deprecated. The first patient in the paper did not undergo the operation and receive the adjuvant radiochemotherapy, although the pathology was clear; 11 months after the operation, the local recurrence occurred. The lesions were insensitive to the salvage chemotherapy and became malignant. Finally, the patient died from the comprehensive recurrence of the chest wall and bone metastases 10 months after the radical resection. For the second patient in the paper, with the malignant AME combined with the infiltrating ductal carcinoma, the adjuvant chemotherapy was carried out; because the hormone receptor of infiltrating ductal carcinoma was positive, the endocrinotherapy has been conducted with the close follow-up visit for 21 months and the general condition of the patient was satisfactory.

The cases with malignant breast AME are rare and such disease is characterized by the strong invasiveness and vulnerability to recurrence and metastasis. Currently, there is no mature clinical experience for reference. Such tumor is inclined to affect the areola area. It is recommended to conduct the simple mastectomy combined with the SLNB due to the low sensitivity of the preoperative imaging diagnosis and difficulty in the pathological diagnosis.

\section{Acknowledgments}

This work was supported by 2015 Beijing Health System Talents Plan (2015-03-008), Beijing Municipal Commission of Health and Family Planning. The authors wish to thank Cancer Research Wales and the Wales Life Science Research Net work (NRN) Network and Ser Cymru for support.

\section{Reference}

1. Tavassoli FA, Devilee P. Tumor pathology and genetics of breast and female reproductive organs. Translated by Cheng Hong, Beijing. People's Medical Publishing House. 2006:99-103.
2. Han B, Mori I, Nakamura M, Wang X, Ozaki T, Nakamura Y, Kakudo K. Myoepithelial carcinoma arising in an adenomyoepithelioma of the breast: case report with immunohistochemical and mutational analysis. Pathol Int. 2006;56(4):211-216.

3. Papazian M, Kalantzis I, Galanopoulos G, Mani I, Tzaida O, Iacovidou I, Ziras N. Malignant myoepithelioma of the breast: A case report and review of the literature. Mol Clin Oncol. 2016;4(5):723-727.

4. Pei F, Zhang HZ, Shi SS, Zhong YX, Wang X, Zhao P. Clinical pathological analysis on two cases with malignant breast adenomyoepithelioma and literature review. Chin J Clin Oncol. 2008;35(19):1097-1099, 1103.

5. Yang Guangzhi, Li Jing, Ding Huaye. Clinical Pathological Observation of Malignant Breast Adenomyoepithelioma. Chinese Journal of Diagnostic Pathology. 2009;16(3):177-180.

6. Chinese anti-cancer association, committee of breast cancer society. Guideline and specification for the diagnosis and treatment of breast cancer. (version 2015). Chinese Anti-Cancer Association. China Oncology. 2015;25(9):692-754.

7. Zhang Zhang, Bu Hong. Adenomyoepithelioma-sourced adenoid cystic carcinoma. Chinese Journal of Clinical and Experimental Pathology. 2014;30(1):61-63.

8. Ahmed AA, Heller DS. Malignant adenomyoepithelioma of the breast with malignant proliferation of epithelial and myoepithelial elements: a case report and review of the literature. Arch Pathol Lab Med. 2000;124(4):632-636.

9. Nadelman CM, Leslie KO, Fishbein MC. "Benign," metastasizing adenomyoepithelioma of the breast: a report of 2 cases. Arch Pathol Lab Med. 2006;130(9):1349-1353.

10. Jones C, Tooze R, Lakhani SR. Malignant adenomyoepithelioma of the breast metastasizing to the liver. Virchows Arch. 2003;442(5):504-506.

11. Bult P, Verwiel JM, Wobbes T, Kooy-Smits MM, Biert J, Holland R. Malignant adenomyoepithelioma of the breast with metastasis in the thyroid gland 12 years after excision of the primary tumor. Case report and review of the literature. Virchows Arch. 2000;436(2):158-166. 